\title{
Limitations of Immunotherapeutic Approaches for Cancer Treatment
}

ISSN: 2637-773X

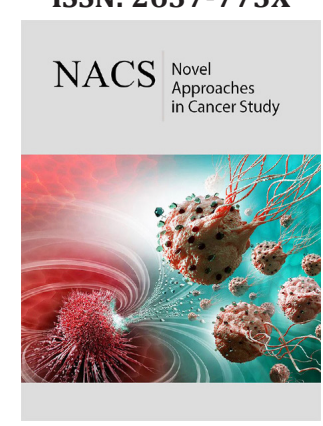

*Corresponding author: Daniel Amsterdam, Emeritus Professor, Departments of Microbiology, Immunology, Pathology and Medicine, Buffalo, New York, USA

Submission: 眥 May 20, 2019

Published: 䟧June 11, 2019

Volume 2 - Issue 5

How to cite this article: Amsterdam D, Ostrov BE. Limitations of Immunotherapeutic Approaches for Cancer Treatment. Nov Appro in Can Study. 2(5). NACS.000549.2019. DOI: $10.31031 /$ NACS.2019.02.000549

Copyright@ Daniel Amsterdam, This article is distributed under the terms of the Creative Commons Attribution 4.0 International License, which permits unrestricted use and redistribution provided that the original author and source are credited.

\section{Daniel Amsterdam ${ }^{1 *}$ and Barbara E Ostrov²}

${ }^{1}$ Emeritus Professor, Departments of Microbiology, Immunology, Pathology and Medicine, Jacobs School of Medicine and Biomedical Sciences, USA

${ }^{2}$ Endowed Professor and Chair, Department of Pediatrics, Albany Medical College, USA

\section{Mini Review}

Of the multiple approaches to cancer therapy, few are as complex as those regimens that encompass immune-based agents. Immunotherapeutic approaches have been the central focus of medical investigators for the past several years. This advance in oncologic care is highlighted by the recognition of the Nobel commission in awarding the 2018 Nobel prize to Allison and Honjo [1]. Their seminal work concentrated on an immunotherapeutic approach to combatting cancers via immune checkpoint receptors' enhancement of the adaptive immune system. A previous review emphasized the potential role of checkpoint inhibitors in the treatment and cure of HIV also through enhancement of adaptive immune function to counter this virus [2].

Immunotherapy with immune checkpoint blockade enhances the response to combatting cancers and acts through boosting the adaptive immune system. It is generally recognized that $\mathrm{T}$ cells possess the capacity to protect from tumor development, in addition to their role in control of infectious diseases. Through immune checkpoint restriction and blocking activity of negative regulators of $\mathrm{T}$ cell activation, cancer control is primarily achieved via actions that block programmed cell death 1 (PD1), cytotoxic T lymphocyte associated antigen 4 (CTLA4) and lymphocyte activation gene 3 (LAG 3).

The success of early treatment trials with the immune checkpoint inhibitors (ICI), CTLA-4 and PD-1, have led to the development (and FDA approval) of several monoclonal antibody medicines. While such novel immunotherapeutic advances have opened new avenues of treatment, there are limitations to these approaches. Failure to respond to these therapies and/or toxicities associated with these agents is the most important limitations of immunotherapies. Cancers may advance, persist and metastasize while undergoing treatment with these agents even in the presence of tumor-infiltrating lymphocytes (TILs), i.e., $\mathrm{T}$ cells with tumor cell-killing capabilities. The absence of response may stem from the lack of immune control due to exhaustion or incomplete reactivity of TILs. This results as a consequence of continued antigen exposure, incomplete expression of rejection by tumor cells, hypoxia, and/or lack of nutrients or substrates in the tumor microenvironment (TME). Studies to understand under-responsiveness to such therapies have uncovered mechanisms by which allocation of metabolic resources supports host defenses. Naïve and memory $\mathrm{T}$ cells rely on catabolic metabolism, (energy-generating processes), whereas effector T cells and macrophages stimulated via Toll-like receptors participate in glycolysis and anabolic metabolism [3].

Additional mechanisms of under-response to immunotherapeutic agents also include hypo-responsiveness of tumor antigen-specific TILs as a consequence of nutrient deficiency and/or suppressive mechanisms in the TME that can be diminished or overcome by modulating the concentration of extracellular potassium in the TME. Vodnala et al. [4] have shown that potassium concentration in the TME is a measure of the dysfunction and stemness of CD8 ${ }^{+}$TILs. Thus, when T cells in the TME are under metabolic constraints their diminished activity can lead to cancer progression, even in the setting of tumors that were previously responsive to immunotherapies [3]. 
Multiple additional factors play a role in patient outcomes using immunotherapeutic approaches. Outcomes can be confounded by variables such as the patient's gut microbiome, diet and potential for infectious or autoimmune complications $[5,6]$. Autoimmune and immune-related toxicities may reflect that mechanisms leading to optimal treatment responses in cancer immunotherapeutics are in direct opposition to the immunologic effects that perpetuate autoimmunity. In the former case, ICI block negative costimulation, whereas in the treatment of autoimmune disease, negative costimulation is promoted. Even in the setting of therapeutic successes, significant toxicity and adverse autoimmune and infectious events have been reported with ICIs [6,7]. Thus far, the benefits of immunotherapeutics in cancer treatment seem to outweigh the risks however this field is young and the full spectrum of unintended consequences of these therapies remain to be uncovered.

\section{References}

1. Zang X (2018) 2018 Nobel Prize in medicine awarded to cancer immunotherapy: Immune checkpoint blockade -A personal account. Genes Dis 5(4): 302-303.
2. Amsterdam D (2015) Immunotherapeutic approaches for the control and eradication of HIV. Immunol Invest 44(8): 719-730.

3. Buck MD, Sowell RT, Kaech SM, Pearce EL (2017) Metabolic instruction of immunity. Cell 169(4): 570-586.

4. Vodnala SK, Eil R, Kishton RJ, Sukumar M, Yamamoto TN, et al. (2019) $\mathrm{T}$ cell stemness and dysfunction in tumors are triggered by a common mechanism. Science Mar 29: 363(6434).

5. Brestoff JR, Artis D (2013) Commensal bacteria at the interface of host metabolism and the immune system. Nat Immunol 14(7): 676-684.

6. Cappelli LC, Gutierrez AK, Bingham CO, Shah AA (2017) Rheumatic and musculoskeletal immune-related adverse events due to immune checkpoint inhibitors: A systematic review of the literature. Arthritis Care Res (Hoboken) 69(11): 1751-1763.

7. Benfaremo D, Manfredi L, Luchetti MM, Gabrielli A (2018) Musculoskeletal and rheumatic diseases induced by immune checkpoint inhibitors: a review of the literature. Curr Drug Saf 13(3): 150-164. 